\title{
Construction of Regular Developable Bézier Patches
}

\author{
Kusno \\ Mathematics Department, University of Jember, Jawa Timur 68121, Indonesia; kusno.fmipa@unej.ac.id; \\ Tel.: +62-812-348-0344
}

Received: 3 December 2018; Accepted: 25 December 2018; Published: 2 January 2019

\begin{abstract}
The modeling of developable surfaces is considered a very important application in plat-metal-based industries. Relating to the purpose, this discussion aims to obtain some formulas for constructing the regular developable Bézier patches, in which each boundary curve must be laid in two parallel planes. The results as follows: We find some formulas of the equation systems that are described by the constant, linear, and quadratic control parameters of the regular developable Bézier patches criteria. The new approach is numerically tested for constructing the regular developable Bézier patches, in which their boundary curves are defined, respectively, by the combination of four, five, and six degrees.
\end{abstract}

Keywords: regular; developable Bézier patches; control parameter

\section{Introduction}

Some methods for designing the developable surfaces have been reported. Aumann [1] defined a developable Bézier patch with two boundary curves (directrices). They are restricted to lie in parallel planes, their projection in the $x-y$ plane is a rectangle, and the tangent vectors to each ruling are parallel. Frey and Bindschadler [2] extended the work of Aumann by generalizing the degree of the directrices. Via linear and quadratic form of regularity condition for Bézier developable, they can generate developable Bézier strip patches that allow their projections in the $x-y$ plane to be trapezoids. In general, the method is simple and easy to use in modeling. However, the method to find the Bézier control points of the equations system is still not stable and deterministic. Chu and Séquin [3] and Aumann [4] developed a technique to determine the geometric design of developable Bézier surfaces based on the de Casteljau algorithm. Chalfant [5] proposed a method to construct a B-spline developable surface bounded by two directrices that lie on a parallel plane. Zhao and Wang [6] presented an approach to design a developable surface in the algebraic form using the surface pencil passing through a given curve. They derived the necessary and sufficient conditions for a surface to be developable by representing the surface with the combination of the given curve, and the three vectors decomposed along the directions of the Serret-Frenet frame. Meanwhile, Al-Ghefari and Abdel [7] presented an approach for designing a developable surface, possessing a given curve as the geodesic of it by analyzing the necessary and sufficient conditions when the resulting developable surface is a cylinder, cone, or tangent surface. Based on results from the optimal control of linear systems with quadratic criteria, Park et al. [8] introduced a mathematical framework for constructing developable surfaces. From a set of rulings, they analyze the existence of base curves, such that the resulting surface is developable. Then, they proposed a class of objective functions that are based on various physical criteria for constructing the base curve. In the context of defining developable approximation surfaces, Elber [9] presented an approximation method that trims the surface using isolines and interpolates each trimmed piece by ruled patches. Sun and Fiume [10] introduced a new modeling system that can be used to create developable surfaces from scratch. They divided a surface to be modeled into several pieces, then defined the shape of each surface piece with a generalized cone. 
After that, they constructed separately each of 3D surface piece using piecewise continuous curves of the cross-section of the cones, then stitched together the surface pieces to construct the desired developable surface. Meanwhile, $\mathrm{Xu}$ et al. [11] presented a novel approach to constructing minimal surface from a given boundary by applying quasi-harmonic Bézier approximation and quasi-harmonic mask methods. Then, $\mathrm{Xu}$ et al. [12] proposed a general framework for constructing IGA-suitable planar B-spline parameterizations from given complex CAD boundaries. This method needs some complex operations to obtain the type surface, e.g., an operation of Bézier extraction and subdivision that are performed on each boundary curve to generate a planar parameterization, a robust planar domain partition framework, quadrangulation, and a local optimization process. A different approach of the methods is presented by Bodduluri and Ravani [13], Pottmann and Farin [14], and Pottmann and Wallner [15]. They constructed the developable surfaces in terms of plane geometry using the concept of duality between points and planes in a 3D projective space. Mathematically, this method is interesting, but it may be less practical in the design of 3D shapes. Hu et al. [16] designed the generalized developable H-Bézier surfaces using control planes with generalized H-Bézier basis functions. Based on the Bodduluri and Ravani idea, Hu et al. [17] developed this method to define the developable Bézier-like surfaces with Bernstein-like basis functions of the multiple shape parameters. The shapes of both type surfaces can be adjusted by altering the values of the shape parameters in that basis functions. Choosing some different values of these shape parameters will produce, respectively, a family of developable H-Bézier and Bézier-like surfaces, without changing the characteristics of Bézier surfaces. The introduced methods generally have some of the following restrictions: The characteristic equations for constructing developable surfaces cannot be solved easily, the planar boundary curves of low degree are more useful for designing developable surfaces, and it is difficult to determine the vertex for a cone and the edge of regression for a tangent surface. To avoid these disadvantages, we propose a new method for designing regular developable Bézier patches, based on the work of Frey and Bindschadler in terms of the projections of the patches in the $x-y$ plane as trapezoids, and their boundary curves to be defined by the combination of four, five, and six degrees.

The paper is organized in the following sections: In the first section, we discuss condition and regularity of the developable Bézier patches, which are supported by two parallel planes. In the second, we talk about the construction of the regular developable Bézier patches models that are defined by two curves planes from the combination of four, five, and six degrees. Finally, the results will be summarized in the conclusion section.

\section{Mathematical Formulations of the Regular Developable Bézier Patches}

The mathematical formulations of the regular developable patches in the algebraic form and Bézier expression are defined as follows:

Definition 1. Ruled surface $\mathbf{S}(u, v)$ of class $C^{n \geq 1}$ is parameterized surface

$$
\mathbf{S}(u, v) \in I \times R \rightarrow \mathbf{f}(u)+v \mathbf{g}(u)
$$

with $\mathbf{f}: I \rightarrow R^{3}$ and $\mathbf{g}: I \rightarrow R^{3}$ of class $\mathbf{C}^{n}$ in $I$ and $\mathbf{o} \notin \mathbf{g}(I)$.

Here, $(I, \mathbf{f})$ is called the directrix curve and $\mathbf{g}(u)$ in the lines form is called the generatrix.

Definition 2. The ruled surface $\mathbf{S}(u, v)=\mathbf{f}(u)+v \mathbf{g}(u)$ is developable, if all $u \in I$ the vectors $\left[\mathbf{g}^{\prime}(u), \mathbf{f}^{\prime}(u), \mathbf{g}(u)\right]$ are coplanar, namely there exists unique real scalars $\delta(u)$ and $\sigma(u)$ such that

$$
\mathbf{g}^{\prime}(u)=\delta(u) \mathbf{f}^{\prime}(u)+\sigma(u) \mathbf{g}(u)
$$


Definition 3. Developable Bézier surface is developable surface that can be constructed by two Bézier curves $\mathbf{C}_{1}(u)$ and $\mathbf{C}_{2}(u)$ in the form

$$
\begin{gathered}
\mathbf{S}(u, v)=\mathbf{C}_{1}(u)+v \mathbf{g}(u) \text { with } \mathbf{g}(u)=\mathbf{C}_{2}(u)-\mathbf{C}_{1}(u) \\
\text { or } \\
\mathbf{S}(u, v)=(1-v) \mathbf{C}_{1}(u)+v \mathbf{C}_{2}(u) \text { where } 0 \leq u, v \leq 1 .
\end{gathered}
$$

The Bézier curves $\mathbf{C}_{1}(u)$ and $\mathbf{C}_{2}(u)$ are defined by the form $\mathbf{C}(u)=\sum_{i=0}^{n} \mathbf{b}_{i} B_{i}^{n}(u)$ with $B_{i}^{n}(u)=C_{i}^{n} \cdot(1-u)^{n-i} \cdot u^{i}$ and $C_{i}^{n}=\frac{n !}{i ! .(n-i) !}$.

Definition 2 implies that the ruled Bézier patch $\mathbf{S}(u, v)$ is developable, if the vectors $\mathbf{g}^{\prime}(u)$, $\mathbf{C}_{1}{ }^{\prime}(u)$ and $\mathbf{g}(u)$ are coplanar. This means that it exists two real scalars $\alpha(u)$ and $\sigma(u)$ such that

$$
\begin{gathered}
\mathbf{g}^{\prime}(u)=\alpha(u) \mathbf{C}_{1}^{\prime}(u)+\sigma(u) \mathbf{g}(u) \\
\text { or } \\
\mathbf{C}_{2}^{\prime}(u)=[1+\alpha(u)] \mathbf{C}_{1}^{\prime}(u)+\sigma(u) \mathbf{g}(u)=\rho(u) \mathbf{C}_{1}^{\prime}(u)+\sigma(u) \mathbf{g}(u) .
\end{gathered}
$$

If $\mathrm{C}_{1}(u)$ and $\mathrm{C}_{2}(u)$ are to be conditioned respectively in two parallel planes $\left[\Psi_{1}, \Psi_{2}\right]$, the developable condition (1) can be simplified as

$$
\mathbf{C}^{\prime}{ }_{2}(u)=\rho(u) \mathbf{C}_{1}^{\prime}(u)
$$

Meanwhile for its regular condition, Frey [2] and Kusno [18] state that the developable Bézier patch $\mathbf{S}(u, v)$ is defined by two curves $\left[\mathbf{C}_{1}(u), \mathbf{C}_{2}(u)\right]$ respectively on two parallel planes $\left[\Psi_{1}, \Psi_{2}\right]$ are regular, if the choice of the real scalar $\rho(u)>0$.

\section{Construction of the Regular Developable Bézier Patches}

Mathematically, one aspect of the most exciting properties of developable surfaces is their ability to be laid flat on a plane without stretching, tearing, or deforming. So, studies about the developable surfaces are considered very important in application in plat-metal-based industries, including ship hulls, aircrafts industries, trains, and automobile parts, such as upholstery and body panels $[2,8,9]$. For that purpose, each small surface section (patch) of those objects, generally, must be modeled by their two boundary curves $C_{1}(u)$ and $C_{2}(u)$ that respectively lie in the plane $\Psi_{1}$ parallel to the plane $\Psi_{2}$. The problem that will be discussed here is how to construct the developable Bézier patches by using both curves.

Consider the regular developable Bézier patches that are defined by two curves $\mathbf{C}_{1}(u)$ and $\mathbf{C}_{2}(u)$ of degree $n$ and $m$, respectively. We note it as $[\operatorname{RDBP}(n, m)]$ and the curves $\mathbf{C}_{1}(u)$ and $\mathbf{C}_{2}(u)$ are expressed as

$$
\mathbf{C}_{1}(u)=\sum_{i=0}^{n} \mathbf{p}_{i} B_{i}^{n}(u) \text { and } \mathbf{C}_{2}(u)=\sum_{i=0}^{m} \mathbf{q}_{i} B_{i}^{m}(u) .
$$

Then, we evaluate some different value of control parameter $\rho(u)$ in Equation (2) that can affect and modify the shape of the modeled regular developable Bézier patches.

\subsection{Case: $\rho(u)$ Constant}

We set two Bézier curves $\mathbf{C}_{1}(u)$ and $\mathbf{C}_{2}(u)$ of the same degree $n$, and the scalar $\rho(u)$ is positive constant, i.e., $\rho(u)=\alpha \in R^{+}$. From the condition (2), we have

$$
\sum_{i=0}^{n-1}\left[\left(\mathbf{q}_{i}-\mathbf{q}_{i+1}\right)+\alpha \cdot\left(\mathbf{p}_{i+1}-\mathbf{p}_{i}\right)\right] B_{i}^{n-1}(u)=\mathbf{0} .
$$


Because of $i=0, \ldots,(n-1)$, the polynomials $B_{i}^{n-1}(u)$ are not zero, then

$$
\left[\left(\mathbf{q}_{i}-\mathbf{q}_{i+1}\right)+\alpha \cdot\left(\mathbf{p}_{i+1}-\mathbf{p}_{i}\right)\right]=\mathbf{0}
$$

for all $i=0, \ldots,(n-1)$. When we add those equations, we will find an equation of the Bézier polygon control points of the curves $\mathbf{C}_{1}(u)$ and $\mathbf{C}_{2}(u)$ as follows

$$
\left[\left(\mathbf{q}_{n}-\mathbf{q}_{0}\right)=\alpha \cdot\left(\mathbf{p}_{n}-\mathbf{p}_{0}\right)\right]
$$

So, to construct a regular developable Bézier patch which is supported by two curves $\mathrm{C}_{1}(u)$ and $\mathrm{C}_{2}(u)$ of degree $n$ and conditioned by $\rho(u)$ positive constant, we note as $[\operatorname{RDBP}(n, n)]_{C}$, must verify the Equations (4) and (5), namely

1. The two vectors parallel $\left(\mathbf{q}_{n}-\mathbf{q}_{0}\right)$ and $\left(\mathbf{p}_{n}-\mathbf{p}_{0}\right)$ must be in the same direction to determine $\alpha$ value;

2. Every $i=0, \ldots, n-1)$ the vector $\left(\mathbf{q}_{i+1}-\mathbf{q}_{i}\right)$ and $\left(\mathbf{p}_{i+1}-\mathbf{p}_{i}\right)$ must be parallel and proportional to $\alpha$.

In relation to treating the geometric continuity order 1 between two adjacent joined patches, in Equation (4), it is necessary to set four boundary control points $\left[\mathbf{p}_{0}, \mathbf{q}_{0}, \mathbf{p}_{n}, \mathbf{q}_{n}\right]$ and two control points $\left[\mathbf{p}_{1}, \mathbf{p}_{n-1}\right]$ of the Bézier curve $\mathbf{C}_{1}(u)$ that are used as the fixed data points. Because of the practical reasons, we are interested in the construction of $[\operatorname{RDBP}(4,4)]_{C}$ and $[\operatorname{RDBP}(6,6)]_{C}$ based on those constrains.

Consider $[\operatorname{RDBP}(4,4)]_{C}$ and from the constrains (4) and (5) for $i=0, \ldots, 3$ we find

$$
\begin{gathered}
{\left[\left(\mathbf{q}_{0}-\mathbf{q}_{4}\right)+\alpha_{[4]} \cdot\left(\mathbf{p}_{4}-\mathbf{p}_{0}\right)\right]=\mathbf{0}} \\
\alpha_{[4]}=\frac{\left\|\left(\mathbf{q}_{4}-\mathbf{q}_{0}\right)\right\|}{\left\|\left(\mathbf{p}_{4}-\mathbf{p}_{0}\right)\right\|} .
\end{gathered}
$$

On the other hand, from $[\operatorname{RDBP}(6,6)]_{C}$ and the constraints (4) and (5) for $i=0, \ldots, 5$ we find

$$
\begin{gathered}
{\left[\left(\mathbf{q}_{0}-\mathbf{q}_{6}\right)+\alpha_{[6]} \cdot\left(\mathbf{p}_{6}-\mathbf{p}_{0}\right)\right]=\mathbf{0}} \\
\alpha_{[6]}=\frac{\left\|\left(\mathbf{q}_{6}-\mathbf{q}_{0}\right)\right\|}{\left\|\left(\mathbf{p}_{6}-\mathbf{p}_{0}\right)\right\|} .
\end{gathered}
$$

In order to obtain an unique solution of the equation system, we set $\left[\mathbf{p}_{0}, \mathbf{p}_{1}, \mathbf{p}_{3}, \mathbf{p}_{4}, \mathbf{q}_{0}, \mathbf{q}_{4}\right]$ for $[\operatorname{RDBP}(4,4)]_{C}$ and $\left[\mathbf{p}_{0}, \mathbf{p}_{1}, \mathbf{p}_{5}, \mathbf{p}_{6}, \mathbf{q}_{0}, \mathbf{q}_{2}, \mathbf{q}_{4}, \mathbf{q}_{6}\right]$ for $[\operatorname{RDBP}(6,6)]_{C}$ as shown in Figure $1 \mathrm{a}, \mathrm{b}$ in black points, then from Equations (6b) and ( $7 b)$ to the system (4), we find respectively four equations to calculate $\left[\mathbf{q}_{1}, \mathbf{q}_{3}, \mathbf{q}_{2}, \mathbf{p}_{2}\right]$ in blue points of control points $[\operatorname{RDBP}(4,4)]_{C}$ such that we obtain

$$
\mathbf{q}_{1}=\mathbf{q}_{0}+\alpha_{[4]} \cdot\left(\mathbf{p}_{1}-\mathbf{p}_{0}\right) ; \mathbf{q}_{3}=\mathbf{q}_{4}-\alpha_{[4]} \cdot\left(\mathbf{p}_{4}-\mathbf{p}_{3}\right) ; \mathbf{q}_{2}=\frac{1}{2}\left(\mathbf{q}_{1}+\mathbf{q}_{3}\right) ; \mathbf{p}_{2}=\frac{1}{2}\left(\mathbf{p}_{1}+\mathbf{p}_{3}\right) ;
$$

and six equations to calculate $\left[\mathbf{q}_{1}, \mathbf{q}_{5}, \mathbf{p}_{2}, \mathbf{p}_{4}, \mathbf{q}_{3}, \mathbf{p}_{3}\right]$ of control points $[\operatorname{RDBP}(6,6)]_{C}$ such that we obtain

$$
\begin{gathered}
\mathbf{q}_{1}=\mathbf{q}_{0}+\alpha_{[6]} \cdot\left(\mathbf{p}_{1}-\mathbf{p}_{0}\right) ; \mathbf{p}_{2}=\frac{1}{\alpha_{[6]}}\left(\mathbf{q}_{2}-\mathbf{q}_{1}+\alpha_{[6]} \cdot \mathbf{p}_{1}\right) ; \mathbf{q}_{5}=\mathbf{q}_{6}-\alpha_{[6]} \cdot\left(\mathbf{p}_{6}-\mathbf{p}_{5}\right) ; \\
\mathbf{p}_{4}=-\frac{1}{\alpha_{[6]}}\left(\mathbf{q}_{5}-\mathbf{q}_{4}-\alpha_{[6]} \cdot \mathbf{p}_{5}\right) ; \mathbf{q}_{3}=\frac{1}{2}\left(\mathbf{q}_{2}+\mathbf{q}_{4}\right) ; \mathbf{p}_{3}=\frac{1}{2}\left(\mathbf{p}_{2}+\mathbf{p}_{4}\right) .
\end{gathered}
$$

Some application examples of the method are shown in Figure 1c,d,g. Meanwhile, Figure 1e,f,h present that along the parameter $u$ of the boundary curves $\mathbf{C}_{1}(u)$ and $\mathbf{C}_{2}(u)$, its tangent vectors and the generatrixes of the patches are coplanar. 
[RDBP $(4,4)] c$ Control Points

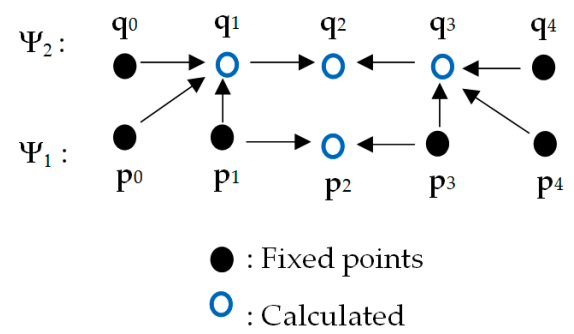

(a)

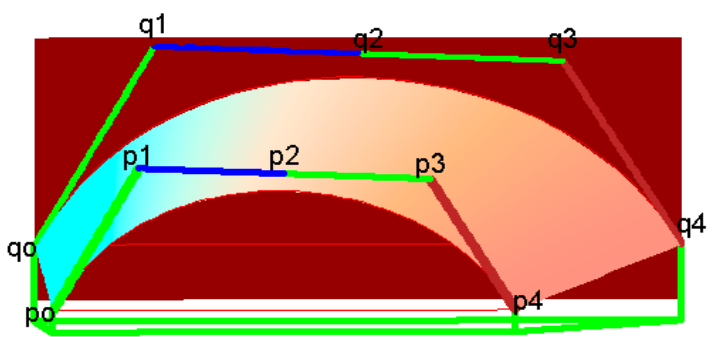

(c)

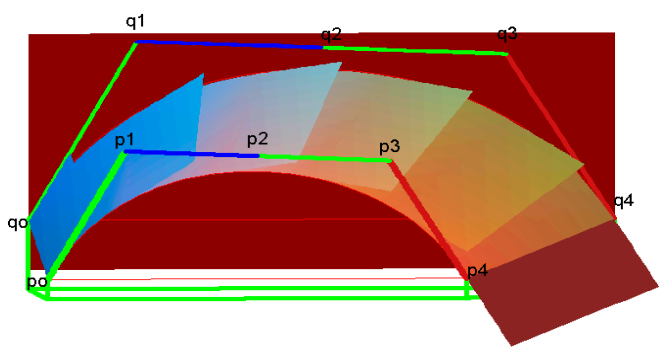

(e)

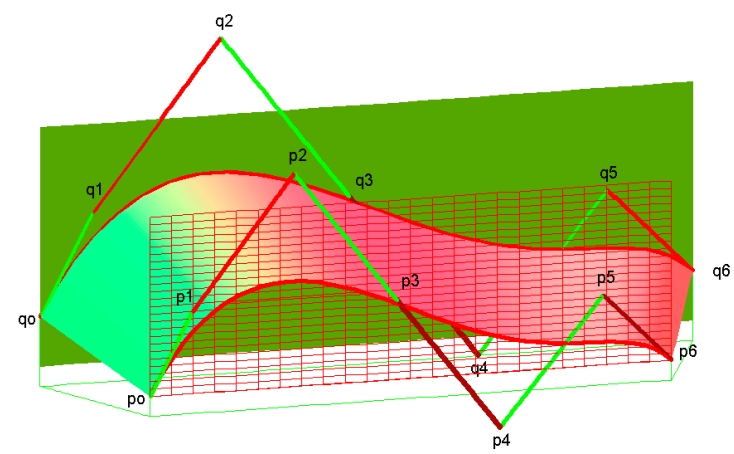

(g)
[RDBP $(6,6)]$ c Control Points

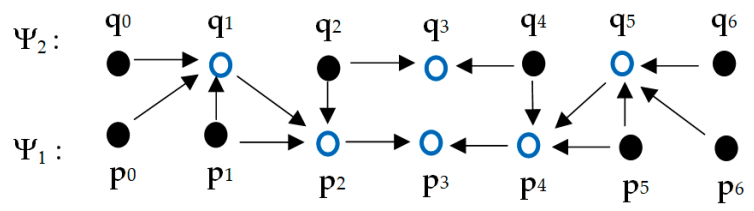

(b)

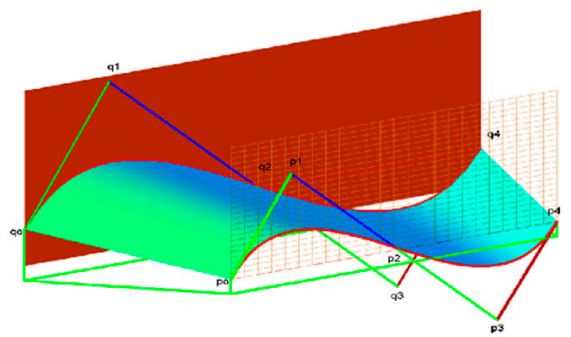

(d)

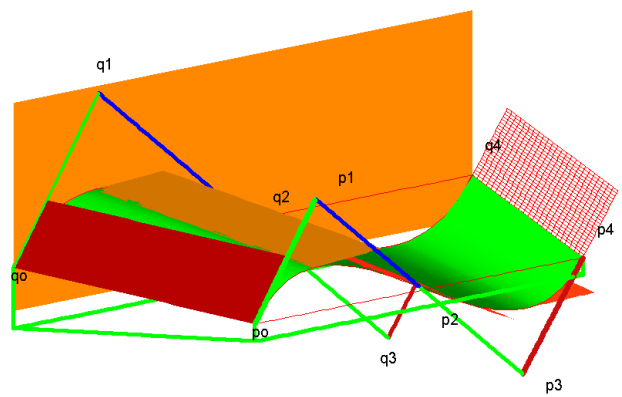

(f)

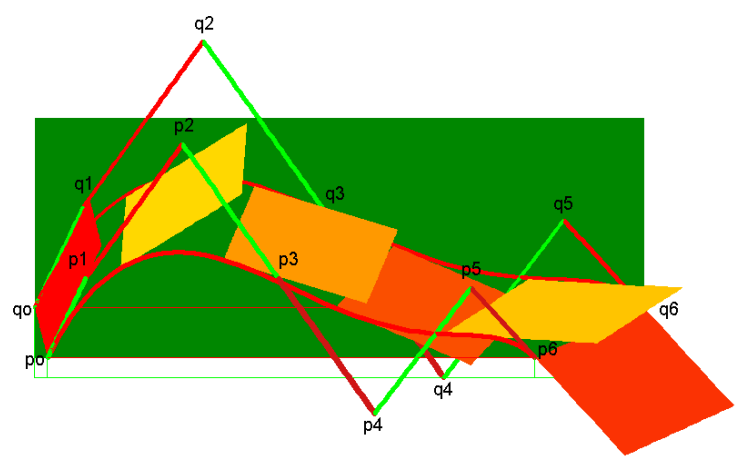

(h)

Figure 1. Construction $[\operatorname{RDBP}(4,4)]_{C}$ and $[\operatorname{RDBP}(6,6)]_{C}$.

It is clear that to construct the $[\operatorname{RDBP}(4,4)]_{C}$, we must first determine the consecutive control points $\left[\mathbf{p}_{0}, \mathbf{p}_{1}, \mathbf{p}_{3}, \mathbf{p}_{4}\right],\left[\mathbf{q}_{0}, \mathbf{q}_{4}\right]$ and $\left(\mathbf{q}_{4}-\mathbf{q}_{0}\right) / /\left(\mathbf{p}_{4}-\mathbf{p}_{0}\right)$. Second, by Equation (6b), we calculate $\alpha_{[4]}$. Finally, the four variable control points $\left[\mathbf{q}_{1}, \mathbf{q}_{3}, \mathbf{q}_{2}, \mathbf{p}_{2}\right]$ can be determined by four equations in the system (4). This method also applies to the $[\operatorname{RDBP}(6,6)]_{C}$.

When the conditions $\left(\mathbf{p}_{n}-\mathbf{p}_{0}\right) / /\left(\mathbf{q}_{n}-\mathbf{q}_{0}\right)$ have been verified, the curves shape $\mathbf{C}_{1}(u)$ and $\mathbf{C}_{2}(u)$ that can define $[\operatorname{RDBP}(4,4)]_{C}$ are fully be affected by the determination of four Bézier control points 
$\left[\mathbf{p}_{0}, \mathbf{p}_{1}, \mathbf{p}_{3}, \mathbf{p}_{4}\right]$ for the curve $\mathbf{C}_{1}(u)$ and two Bézier control points $\left[\mathbf{q}_{0}, \mathbf{q}_{4}\right]$, tangent vector $\left(\mathbf{p}_{1}-\mathbf{p}_{0}\right)$ and $\left(\mathbf{p}_{4}-\mathbf{p}_{3}\right)$ for the curve $\mathbf{C}_{2}(u)$. Figure 1a shows that the Bézier control points $\mathbf{q}_{1}$ and $\mathbf{q}_{3}$ are defined, respectively, by $\left[\mathbf{q}_{0}, \mathbf{p}_{0}, \mathbf{p}_{1}\right]$ and $\left[\mathbf{q}_{4}, \mathbf{p}_{3}, \mathbf{p}_{4}\right]$. The Bézier control points $\mathbf{q}_{2}$ and $\mathbf{p}_{2}$, respectively, depend on the control points $\left[\mathbf{q}_{1}, \mathbf{q}_{3}\right]$ and $\left[\mathbf{p}_{1}, \mathbf{p}_{3}\right]$. By changing the two tangent vectors direction (Figure $1 \mathrm{c}, \mathrm{d}$ ), we can modify the curve shape $\mathbf{C}_{1}(u)$ and $\mathbf{C}_{2}(u)$. On the other hand, the curves shape $\mathbf{C}_{1}(u)$, and $\mathbf{C}_{2}(u)$ of $[\operatorname{RDBP}(6,6)]_{C}$ depend on some Bézier control points of both curves. Figure $1 \mathrm{~b}$ shows that we can elect some Bézier control points of the curve $C_{1}(u)$ and $C_{2}(u)$ respectively in plan $\Psi_{1}$ and $\Psi_{2}$ to obtain the various forms of that curves. Figure $1 \mathrm{~g}$ presents one result example of that method. To ensure that the patches are developable, Figure 1e,f,h show that (at all parameter values, $u$, certainly) its tangent vectors and generatrixes must be coplanar. Based on the result of the discussion, we can conclude that the construction method $[\operatorname{RDBP}(n, n)]_{C}$ can facilitate to model the shape of developable patches.

\subsection{Case: $\rho(u)$ Linear}

Let two Bézier curves, $\mathbf{C}_{1}(u)$ and $\mathbf{C}_{2}(u)$, respectively, of degree $n$ and $n+1$, in two parallel planes [ $\Psi_{1}, \Psi_{2}$ ]. The scalar $\rho(u)=\alpha_{0}(1-u)+\alpha_{1} u$ is a real positive. We have the identity [19]

$$
(1-u)^{k 1} \cdot u^{k 2} \sum_{i=0}^{n-1} \mathbf{a}_{i} B_{i}^{n}(u)=\sum_{i=0}^{n+k 1+k 2}\left[\frac{N_{1} \cdot N_{2}}{D}\right] \mathbf{a}_{i-k 2} B_{i}^{n+k 1+k 2}(u)
$$

where

$N_{1}=\left(n+k_{2}+1-i\right)\left(n+k_{2}+2-i\right) \ldots\left(n+k_{2}+k_{1}-i\right)$ if $k_{1} \geq 1$, if not $1 ;$

$N_{2}=\left(i-k_{2}+1\right)\left(i-k_{2}+2\right) \ldots(i)$, if $k_{2} \geq 1$, if not 1 ;

$D=(n+1)(n+2) \ldots\left(n+k_{1}+k_{2}\right)$, if $\left(k_{1}+k_{2}\right) \geq 1$, if not 1 .

Therefore, from Equations (2) and (8), we have

$$
\sum_{i=0}^{n}\left[(n+1)\left(\mathbf{q}_{i}-\mathbf{q}_{i+1}\right)+\alpha_{0}(n-i)\left(\mathbf{p}_{i+1}-\mathbf{p}_{i}\right)+\alpha_{1}(i)\left(\mathbf{p}_{i}-\mathbf{p}_{i-1}\right)\right] B_{i}^{n-1}(u)=\mathbf{0}
$$

Because of $i=0, \ldots,(n-1)$ the polynomials $B_{i}^{n-1}(u)$ are not zero, this mean that

$$
\left[(n+1)\left(\mathbf{q}_{i}-\mathbf{q}_{i+1}\right)+\alpha_{0}(n-i)\left(\mathbf{p}_{i+1}-\mathbf{p}_{i}\right)+\alpha_{1}(i)\left(\mathbf{p}_{i}-\mathbf{p}_{i-1}\right)\right]=\mathbf{0}
$$

for all $i=0, \ldots, \mathrm{n}$. When we add those equations, we will find an equation of control points of both curves and the centroid point of the Bézier polygon $\mathbf{C}_{1}(u)$ as follows

$$
\left(\mathbf{q}_{n+1}-\mathbf{q}_{0}\right)=\alpha_{0}\left(\mathbf{p}-\mathbf{p}_{0}\right)+\alpha_{1}\left(\mathbf{p}_{n}-\mathbf{p}\right)
$$

and $\mathbf{p}=\frac{1}{n} \sum_{i=0}^{n} \mathbf{p}_{i}$. Thus, to construct the regular developable Bézier patches with the two Bézier curves $\mathbf{C}_{1}(u)$ and $\mathbf{C}_{2}(u)$ of degree $n$ and $n+1$ that lie, respectively, in two parallel planes $\left[\Psi_{1}, \Psi_{2}\right]$ and the parameter $\rho(u)$ is positive linear, that is noted $[\operatorname{RDBP}(n, n+1)]_{L}$, can be summarized as follows:

1. Because $\alpha_{0}$ and $\alpha_{1}$ must be positive, the position of the vector $\left(\mathbf{q}_{n}-\mathbf{q}_{0}\right)$ in Equation (10) must lie on the interior of the angle between two vectors $\left(\mathbf{p}-\mathbf{p}_{0}\right)$ and $\left(\mathbf{p}_{n}-\mathbf{p}\right)$. If the control points $\left[\mathbf{p}_{0}, \mathbf{p}_{n}, \mathbf{q}_{0}, \mathbf{q}_{n+1}\right]$ are fixed, then the selection of the centroid point $\mathbf{p}$ must satisfy the conditions that the direction of the vector $\left(\mathbf{p}-\mathbf{p}_{0}\right)$ and $\left(\mathbf{p}_{n}-\mathbf{p}\right)$ are in the different sides to the vector $\left(\mathbf{q}_{n}-\mathbf{q}_{0}\right)$.

2. The value of $\alpha_{0}$ and $\alpha_{1}$ in Equation (10) can be calculated by

$$
\alpha_{0}=\frac{\left\|\left(\mathbf{q}_{n+1}-\mathbf{q}_{0}\right) \wedge\left(\mathbf{p}_{n}-\mathbf{p}\right)\right\|}{\left\|\left(\mathbf{p}-\mathbf{p}_{0}\right) \wedge\left(\mathbf{p}_{n}-\mathbf{p}\right)\right\|}
$$




$$
\alpha_{1}=\frac{\left\|\left(\mathbf{q}_{n+1}-\mathbf{q}_{0}\right) \wedge\left(\mathbf{p}-\mathbf{p}_{0}\right)\right\|}{\left\|\left(\mathbf{p}_{n}-\mathbf{p}\right) \wedge\left(\mathbf{p}-\mathbf{p}_{0}\right)\right\|} .
$$

3. To find the unique solution of the system (9), we use the strategy as follows. It has to determine $(n+2)$ control points of $(2 n+3)$ control points in the system, such that the number $(n+1)$ calculated control points are the same as the number of these equations, i.e., $(n+1)$ equations. Also, the determinant of its coefficients matrix of the system must be different from zero. In relation to treating the geometric continuity order 1 between two adjacent joined patches, it is necessary that the points $\left[\mathbf{p}_{0}, \mathbf{p}_{1}, \mathbf{p}_{n-1}, \mathbf{p}_{n}, \mathbf{q}_{0}, \mathbf{q}_{n+1}\right]$ are included in the list of the $(n+2)$ fixed points.

Based on these constraints, we then illustrate the construction $[\operatorname{RDBP}(4,5)]_{L}$ and $[\operatorname{RDBP}(5,6)]_{L}$. The resulting sample of this method is visualized in Figures 2-4.

\section{$[\operatorname{RDBP}(4,5)] L$ Control Points}

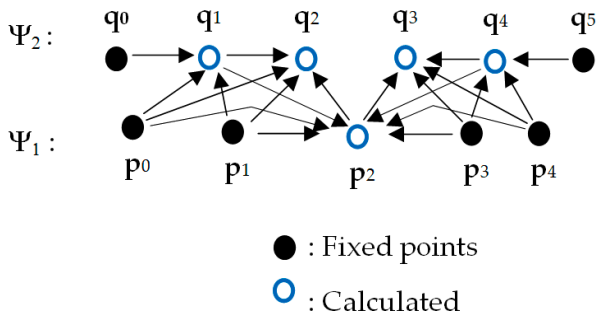

(a)

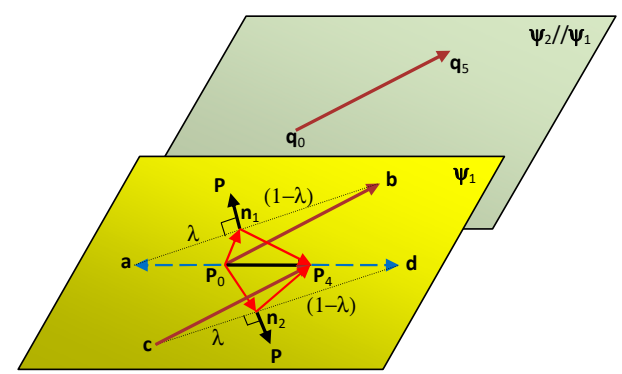

(c)

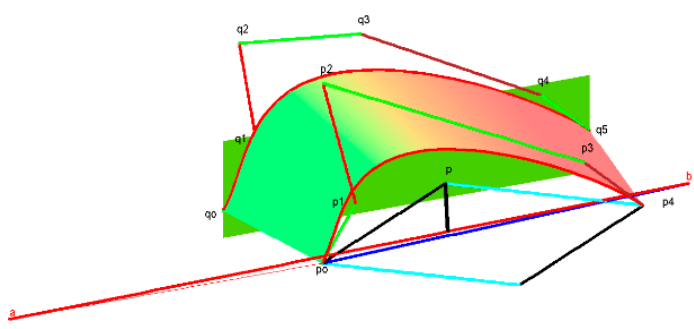

(e)

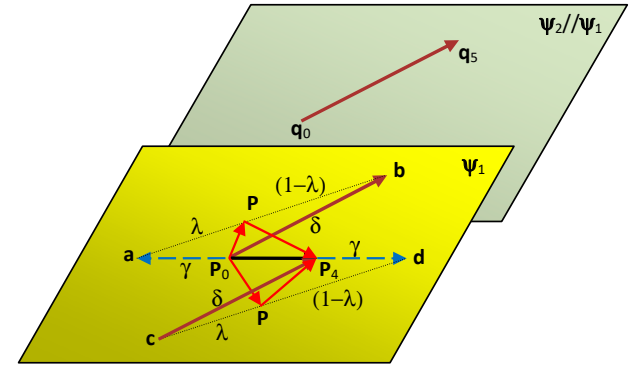

(b)

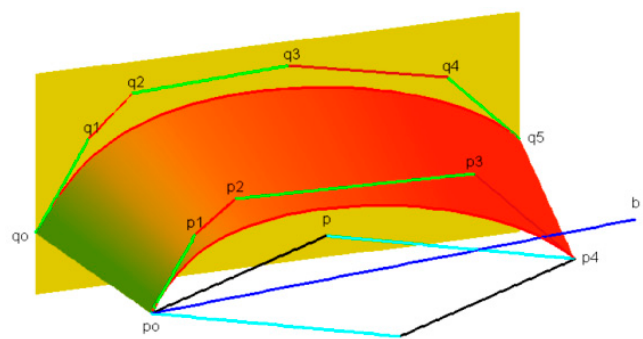

(d)

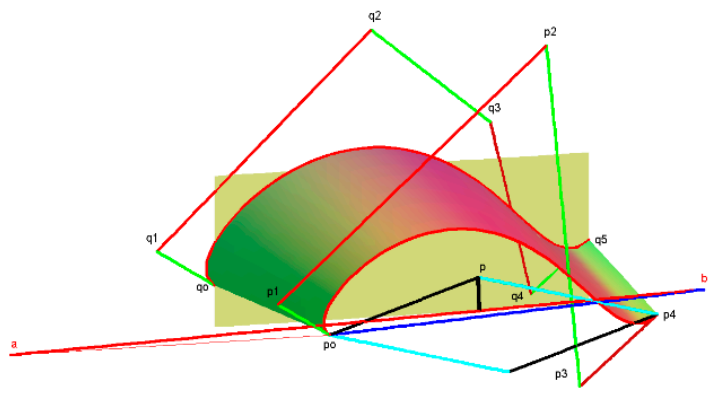

(f)

Figure 2. Setting the Bézier control points and the centroid point $[\operatorname{RDBP}(4,5)]_{L}$. 


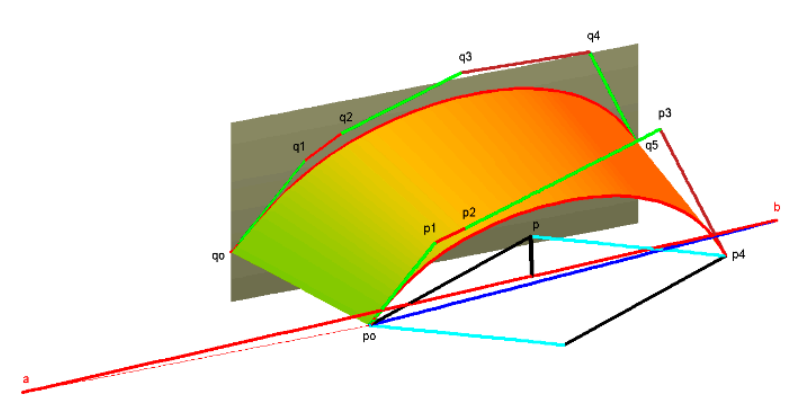

(a)

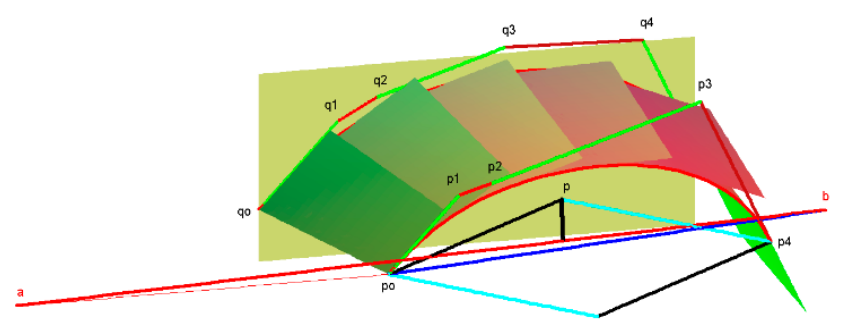

(c)

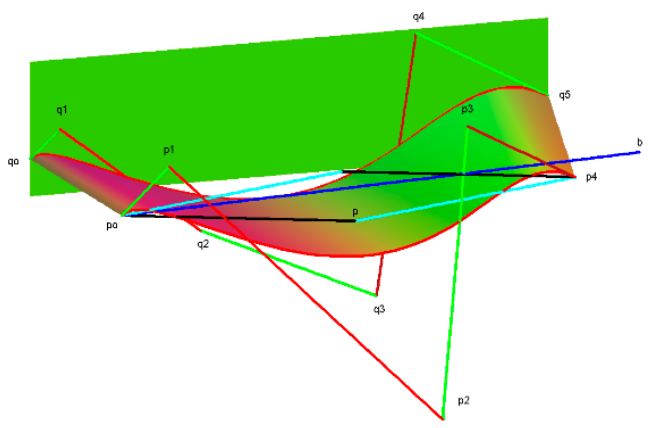

(b)

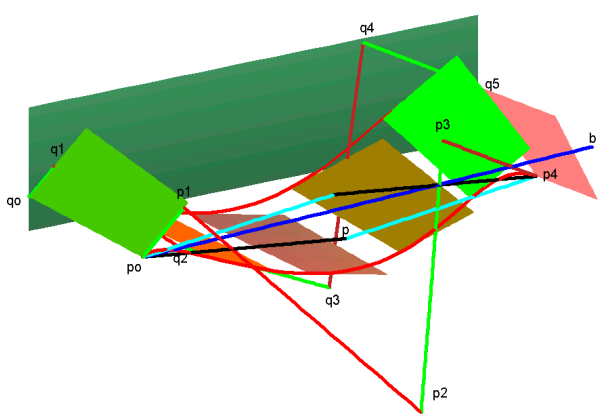

(d)

Figure 3. Construction $[\operatorname{RDBP}(4,5)]_{\mathrm{L}}$.

$[\operatorname{RDBP}(5,6)]_{L}$ Control Points

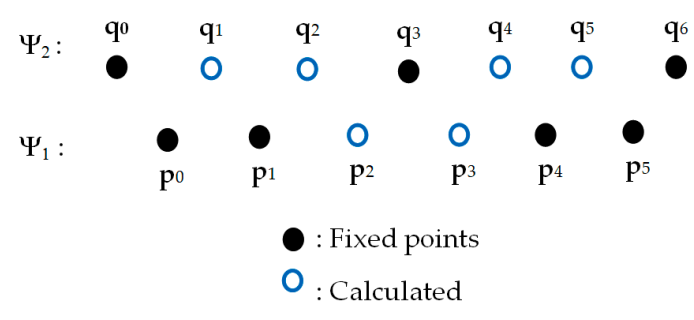

(a)

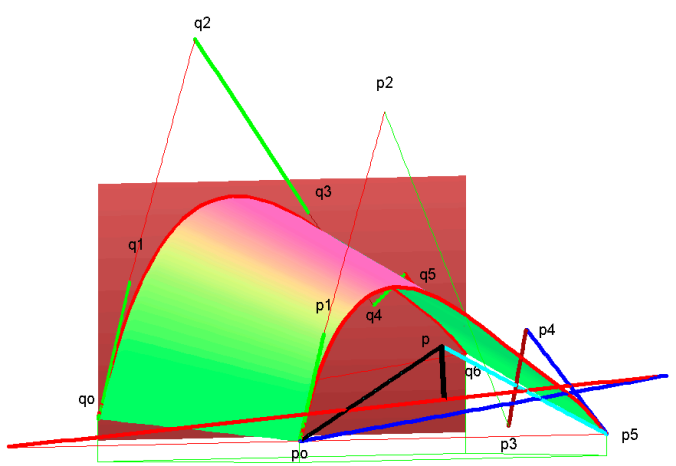

(c)

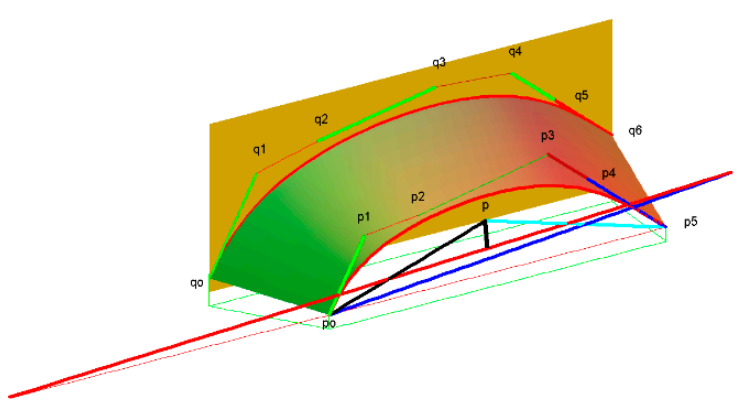

(b)

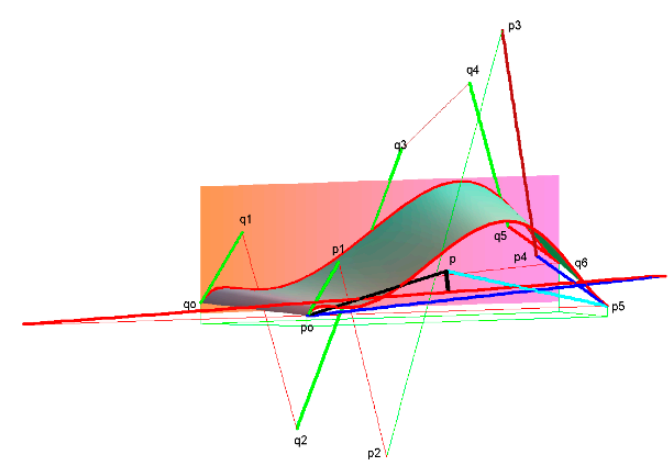

(d)

Figure 4. Construction $[\operatorname{RDBP}(5,6)]_{L}$.

Let $[\operatorname{RDBP}(4,5)]_{L}$ and from Equation (9), for $i=0, \ldots, 4$ we find the control points Equation (10) in the form

$$
\left(\mathbf{q}_{5}-\mathbf{q}_{0}\right)=\alpha_{0}\left(\mathbf{p}-\mathbf{p}_{0}\right)+\alpha_{1}\left(\mathbf{p}_{4}-\mathbf{p}\right) .
$$


In order to obtain an unique solution of the equation system, if the points $\left[\mathbf{p}_{0}, \mathbf{p}_{1}, \mathbf{p}_{3}, \mathbf{p}_{4}, \mathbf{q}_{0}, \mathbf{q}_{5}\right]$ are fixed (Figure 2a) by constraint (1), we can determine the centroid point $\mathbf{p}$ of Bézier polygon of the curve $\mathbf{C}_{1}(u)$, such that the direction of the vectors $\left(\mathbf{p}-\mathbf{p}_{0}\right)$ and $\left(\mathbf{p}_{4}-\mathbf{p}\right)$ are in the different sides to the vector $\left(\mathbf{q}_{5}-\mathbf{q}_{0}\right)$. Clearly, in Figure $2 \mathbf{b}, \mathrm{d}$,e, let in plane $\Psi_{1}$, the vectors $\left(\mathbf{b}-\mathbf{p}_{0}\right)=\left(\mathbf{p}_{4}-\mathbf{c}\right)=\left(\mathbf{q}_{5}-\mathbf{q}_{0}\right)$ and $\left(\mathbf{p}_{4}-\mathbf{p}_{0}\right)=\left(\mathbf{d}-\mathbf{p}_{4}\right)=\left(\mathbf{p}_{0}-\mathbf{a}\right)$. So, to facilitate the modeling patch, one of our options is to put the centroid point $\mathbf{p}$ in position

$$
\mathbf{p}=\lambda\left[\mathbf{P}_{0}+\delta\left(\mathbf{q}_{5}-\mathbf{q}_{0}\right)\right]+(1-\lambda)\left[\mathbf{P}_{0}-\gamma\left(\mathbf{P}_{4}-\mathbf{P}_{0}\right)\right]
$$

or

$$
\mathbf{p}=\lambda\left[\mathbf{P}_{4}+\gamma\left(\mathbf{P}_{4}-\mathbf{P}_{0}\right)\right]+(1-\lambda)\left[\mathbf{P}_{4}-\delta\left(\mathbf{q}_{5}-\mathbf{q}_{0}\right)\right]
$$

with $\lambda \in(0,1)$ and $\delta, \gamma>0$. In the other hand, for $\tau>0$, we can choose $\mathbf{p}$ in other form (Figure 2c)

$$
\mathbf{p}=[\lambda \mathbf{b}+(1-\lambda) \mathbf{a}]+\tau \mathbf{n}_{1} \text { or } \mathbf{p}=[\lambda \mathbf{d}+(1-\lambda) \mathbf{c}]+\tau \mathbf{n}_{2}
$$

with

$$
\mathbf{n}_{1}=\frac{(\mathbf{b}-\mathbf{a}) \wedge\left(\left(\mathbf{b}-\mathbf{p}_{0}\right) \wedge\left(\mathbf{p}_{4}-\mathbf{p}_{0}\right)\right)}{\left\|(\mathbf{b}-\mathbf{a}) \wedge\left(\left(\mathbf{b}-\mathbf{p}_{0}\right) \wedge\left(\mathbf{p}_{4}-\mathbf{p}_{0}\right)\right)\right\|} \text { and } \mathbf{n}_{2}=\frac{(\mathbf{d}-\mathbf{c}) \wedge\left(\left(\mathbf{p}_{4}-\mathbf{p}_{0}\right) \wedge\left(\mathbf{b}-\mathbf{p}_{0}\right)\right)}{\left\|(\mathbf{d}-\mathbf{c}) \wedge\left(\left(\mathbf{p}_{4}-\mathbf{p}_{0}\right) \wedge\left(\mathbf{b}-\mathbf{p}_{0}\right)\right)\right\|}
$$

respectively the unity vectors that are perpendicular to $(\mathbf{b}-\mathbf{a})$ and $(\mathbf{d}-\mathbf{c})$ in $\Psi_{1}$. After that, we calculate $\alpha_{0}$ and $\alpha_{1}$ in Equation (12) that have been formulated in Equation (11). Moreover, from the equation system (9) we have five equations to calculate the five control points $\left[\mathbf{q}_{1}, \mathbf{q}_{4}, \mathbf{p}_{2}, \mathbf{q}_{2}, \mathbf{q}_{3}\right]$ and we will find $\mathbf{q}_{1}=\frac{1}{5}\left[5 \mathbf{q}_{0}+4 \alpha_{0}\left(\mathbf{p}_{1}-\mathbf{p}_{0}\right)\right] ; \mathbf{q}_{4}=\frac{1}{5}\left[-5 \mathbf{q}_{5}+4 \alpha_{1}\left(\mathbf{p}_{4}-\mathbf{p}_{3}\right)\right] ;$ and

$$
\begin{gathered}
5 \mathbf{q}_{2}-3 \alpha_{0} \mathbf{p}_{2}=\left[5 \mathbf{q}_{1}-3 \alpha_{0} \mathbf{p}_{1}+\alpha_{1}\left(\mathbf{p}_{1}-\mathbf{p}_{0}\right)\right]=\mathbf{a}_{1} \\
-5 \mathbf{q}_{2}+5 \mathbf{q}_{3}+\left(2 \alpha_{0}-2 \alpha_{1}\right) \mathbf{p}_{2}=\left[2 \alpha_{0} \mathbf{p}_{3}-2 \alpha_{1} \mathbf{p}_{1}\right]=\mathbf{a}_{2} \\
-5 \mathbf{q}_{3}+3 \alpha_{1} \mathbf{p}_{2}=\left[-5 \mathbf{q}_{4}+\alpha_{0}\left(\mathbf{p}_{4}-\mathbf{p}_{3}\right)+3 \alpha_{1} \mathbf{p}_{3}\right]=\mathbf{a}_{3}
\end{gathered}
$$

The application examples of the method are shown in Figure $2 \mathrm{~d}-\mathrm{f}$.

The Equation (14) have a unique solution if its determinant of the co-efficient matrix is different from zero, i.e.,

$$
\operatorname{det}\left(\begin{array}{ccc}
5 & 0 & -3 \alpha_{0} \\
-5 & 5 & \left(2 \alpha_{0}-2 \alpha_{1}\right) \\
0 & -5 & 3 \alpha_{1}
\end{array}\right) \neq 0
$$

This mean that if $\alpha_{\mathrm{o}} \neq \alpha_{1}$, then the unique solution of Equations (14) are

$$
\mathbf{q}_{2}=\frac{1}{5}\left[\mathbf{a}_{1}+3 \alpha_{0} \mathbf{p}_{2}\right] ; \mathbf{q}_{3}=\frac{1}{5}\left[\mathbf{a}_{1}+\mathbf{a}_{2}+\left(\alpha_{0}+2 \alpha_{1}\right) \mathbf{p}_{2}\right] ; \mathbf{p}_{2}=\frac{1}{\left(\alpha_{1}-\alpha_{0}\right)}\left[\mathbf{a}_{1}+\mathbf{a}_{2}+\mathbf{a}_{3}\right] .
$$

In Figure $3 a, b$, we show the effect of the centroid points $\mathbf{p}$ of the $[\operatorname{RDBP}(4,5)]_{L}$ when they are positioned in above and below of $\left(\mathbf{p}_{4}-\mathbf{p}_{0}\right)$. Meanwhile, Figure $3 c, d$ present that along parameter values $u$ of the boundary curves $\mathbf{C}_{1}(u)$ and $\mathbf{C}_{2}(u)$, tangent vectors and the generatrixes of the patches are coplanar.

In case of the construction $[\operatorname{RDBP}(5,6)]_{L}$, if we determine the control points $\left[\mathbf{p}_{0}, \mathbf{p}_{1}, \mathbf{p}_{4}, \mathbf{p}_{5}, \mathbf{q}_{0}, \mathbf{q}_{3}\right.$, $\mathbf{q}_{6}$ ] are shown in the black points of Figure $4 a$, then from the Equation (10) we will find the equation

$$
\left(\mathbf{q}_{6}-\mathbf{q}_{0}\right)=\alpha_{0}\left(\mathbf{p}-\mathbf{p}_{0}\right)+\alpha_{1}\left(\mathbf{p}_{5}-\mathbf{p}\right)
$$


Determination of the centroid point $\mathbf{p}$ is analogous to $[\operatorname{RDBP}(4,5)]_{L}$. The value of $\alpha_{0}$ and $\alpha_{1}$ can be calculated by the formula (11). The equation system (9) will give six equations for calculating the control points $\left[\mathbf{q}_{1}, \mathbf{q}_{5}, \mathbf{p}_{2}, \mathbf{p}_{3}, \mathbf{q}_{2}, \mathbf{q}_{4}\right]$ and we will obtain $\mathbf{q}_{1}=\mathbf{q}_{0}+\frac{5}{6} \alpha_{0}\left(\mathbf{p}_{1}-\mathbf{p}_{0}\right) ; \mathbf{q}_{5}=\mathbf{q}_{6}-\frac{5}{6} \alpha_{1}\left(\mathbf{p}_{5}-\mathbf{p}_{4}\right)$ and

$$
\begin{gathered}
6 \mathbf{q}_{2}-4 \alpha_{0} \mathbf{p}_{2}=\left[6 \mathbf{q}_{1}-4 \alpha_{0} \mathbf{p}_{1}+\alpha_{1}\left(\mathbf{p}_{1}-\mathbf{p}_{0}\right)\right]=\mathbf{z}_{1} \\
-6 \mathbf{q}_{2}+\left(3 \alpha_{0}-2 \alpha_{1}\right) \mathbf{p}_{2}-3 \alpha_{0} \mathbf{p}_{3}=\left[-6 \mathbf{q}_{3}-2 \alpha_{1} \mathbf{p}_{1}\right]=\mathbf{z}_{2} \\
6 \mathbf{q}_{4}+\left(2 \alpha_{0}-3 \alpha_{1}\right) \mathbf{p}_{3}+3 \alpha_{1} \mathbf{p}_{2}=\left[6 \mathbf{q}_{3}+2 \alpha_{0} \mathbf{p}_{4}\right]=\mathbf{z}_{3} \\
-6 \mathbf{q}_{4}+4 \alpha_{1} \mathbf{p}_{3}=\left[\alpha_{0}\left(\mathbf{p}_{5}-\mathbf{p}_{4}\right)+4 \alpha_{1} \mathbf{p}_{4}-6 \mathbf{q}_{5}\right]=\mathbf{z}_{4} .
\end{gathered}
$$

Determinant of the co-efficient matrix of Equations (17) is $D=-144 \alpha_{1} \alpha_{0}+72 \alpha_{1}^{2}+72 \alpha_{0}^{2}$. So, when $\alpha_{0} \neq \alpha_{1}$, the unique solution of the system is

$$
\begin{gathered}
\mathbf{P}_{2}=\frac{1}{\left[9 \alpha_{0} \alpha_{1}-\left(2 \alpha_{0}+\alpha_{1}\right)\left(\alpha_{0}+2 \alpha_{1}\right)\right]}\left[\left(2 \alpha_{o}+\alpha_{1}\right)\left(\mathbf{z}_{1}+\mathbf{z}_{2}\right)+\left(3 \alpha_{o}\right)\left(\mathbf{z}_{3}+\mathbf{z}_{4}\right)\right] ; \\
\mathbf{P}_{3}=\frac{1}{[2 \alpha o+\alpha 1]}\left[\mathbf{z}_{3}+\mathbf{z}_{4}-3 \alpha_{1} \mathbf{P}_{2}\right] ; \mathbf{q}_{2}=\frac{1}{6}\left[4 \alpha_{0} \mathbf{p}_{2}+\mathbf{z}_{1}\right] ; \mathbf{q}_{4}=\frac{1}{6}\left[4 \alpha_{1} \mathbf{p}_{3}-\mathbf{z}_{4}\right] .
\end{gathered}
$$

Some application examples of the method are shown in the Figure $4 \mathrm{~b}-\mathrm{d}$.

The control points setting to model the shape of the curve $\mathbf{C}_{1}(u)$ and $\mathbf{C}_{2}(u)$ of construction $[\operatorname{RDBP}(n, n+1)]_{L}$ are principally like the construction $[\operatorname{RDBP}(n, n)]_{C}$. However, the condition of two fixed vectors $\left(\mathbf{p}_{n}-\mathbf{p}_{\mathrm{o}}\right)$ and $\left(\mathbf{q}_{n+1}-\mathbf{q}_{0}\right)$ of the forth boundary control points $\left[\mathbf{p}_{o}, \mathbf{p}_{n}, \mathbf{q}_{0}, \mathbf{q}_{n+1}\right]$ must not be parallel. Also, we can change the centroid point $\mathbf{P}$ in position to the left, right, up, or down to modify the surface shape of the wanted patches as shown in the examples Figure $2 d-f$, Figure $3 a, b$, and Figure $4 b-d$. To ensure that the patches are developable, Figure $3 c, d$ presents the tangent vectors and generatrixes of the patches that must be coplanar (at all parameter values, $u$, certainly).

\subsection{Case: $\rho(u)$ Quadratic}

Let two Bézier curves $\mathbf{C}_{1}(u)$ and $\mathbf{C}_{2}(u)$, respectively, of degree $n$, and $n+2$ positioning in two parallel planes $\left[\Psi_{1}, \Psi_{2}\right]$. We evaluate the regular developable Bézier patches construction if $\rho(u)>0$ in the form $\rho(u)=\left[\alpha_{0}(1-u)+2 \alpha_{1}(1-u) u+\alpha_{2} u\right]$. Thus, from the Equations (2) and (8), we find

$$
\begin{gathered}
\sum_{i=0}^{n+1}\left[(n+1)(n+2)\left(\mathbf{q}_{i}-\mathbf{q}_{i+1}\right)+\alpha_{0}(n-i)(n+1-i)\left(\mathbf{p}_{i+1}-\mathbf{p}_{i}\right)+\right. \\
\left(\alpha_{0}+2 \alpha_{1}+\alpha_{2}\right)(i)(n+1-i)\left(\mathbf{p}_{i}-\mathbf{p}_{i-1}+\alpha_{2}(i)(i-1)\left(\mathbf{p}_{i-1}-\mathbf{p}_{i-2}\right)\right] B_{i}^{n+1}(u)=\mathbf{0} .
\end{gathered}
$$

So, we have the control points equation system

$$
\begin{gathered}
{\left[(n+1)(n+2)\left(\mathbf{q}_{i}-\mathbf{q}_{i+1}\right)+\alpha_{0}(n-i)(n+1-i)\left(\mathbf{p}_{i+1}-\mathbf{p}_{i}\right)+\right.} \\
\left.\left(\alpha_{0}+2 \alpha_{1}+\alpha_{2}\right)(i)(n+1-i)\left(\mathbf{p}_{i}-\mathbf{p}_{i-1}\right)+\alpha_{2}(i)(i-1)\left(\mathbf{p}_{i-1}-\mathbf{p}_{i-2}\right)\right]=\mathbf{0}
\end{gathered}
$$

for all $i=0, \ldots, n+1$. When we add those equations, we will find the control points and the centroid point equation of the Bézier polygon of the curves $\mathbf{C}_{1}(u)$ and $\mathbf{C}_{2}(u)$ as follows

$$
\left(\mathbf{q}_{n+2}-\mathbf{q}_{0}\right)=\alpha_{0}\left(\mathbf{p}-\mathbf{p}_{0}\right)+\alpha_{2}\left(\mathbf{p}_{n}-\mathbf{p}\right)+\alpha_{1}\left[\frac{4}{15}\left(\mathbf{p}_{n}-\mathbf{p}_{0}\right)+\frac{2}{15}\left(\mathbf{p}_{n-1}-\mathbf{p}_{1}\right)\right]
$$

or

$$
\left[\left(\mathbf{q}_{n+2}-\mathbf{q}_{0}\right)-\alpha_{1} \mathbf{v}\right]=\alpha_{0}\left(\mathbf{p}-\mathbf{p}_{0}\right)+\alpha_{2}\left(\mathbf{p}_{n}-\mathbf{p}\right)
$$

where $\mathbf{v}=\left[\frac{4}{15}\left(\mathbf{p}_{n}-\mathbf{p}_{0}\right)+\frac{2}{15}\left(\mathbf{p}_{n-1}-\mathbf{p}_{1}\right)\right]$. If $\alpha_{0}, \alpha_{2}>0$, then the vector $\left[\left(\mathbf{q}_{n+2}-\mathbf{q}_{0}\right)-\alpha_{1} \mathbf{v}\right]$ must be positive. To ensure this condition, we limit the value $\alpha_{1}$ in the interval $-w<\alpha_{1}<w$ with $w=\frac{\left\|\mathbf{q}_{n+2}-\mathbf{q}_{0}\right\|}{\|\mathbf{v}\|}$. 
Thus, to construct the regular developable Bézier patches with curves $\mathbf{C}_{1}(u)$ and $\mathbf{C}_{2}(u)$, respectively, of degree $n, n+2$ in two parallel planes $\left[\Psi_{1}, \Psi_{2}\right]$, and the parameter $\rho(u)=\left[\alpha_{0}(1-u)+2 \alpha_{1}(1-u) u+\right.$ $\alpha_{2} u$ ] positive quadratic that is noted $[\operatorname{RDBP}(n, n+2)]_{Q}$, can be summarized as follows:

1. Because $\alpha_{0}$ and $\alpha_{1}$ must be positive, the election $\alpha_{1}$ in Equation (19) must verify the expression (20) or the vector $\left[\left(\mathbf{q}_{n+2}-\mathbf{q}_{0}\right)-\alpha_{1} \mathbf{v}\right]$ in Equation (19) must lie on the interior of the angle between two vectors $\left(\mathbf{p}-\mathbf{p}_{0}\right)$ and $\left(\mathbf{p}_{n}-\mathbf{p}\right)$.

2. Calculation of the value $\alpha_{0}$ and $\alpha_{1}$ in Equation (19) is like the formula (11).

3. To find the exact solution of the system (18), we have to determine $(n+2)$ control points of $(2 n+4)$ control points in the system, such that the number $(n+2)$ calculated control points are the same as the number of these Equation (18). The determinant of its coefficients matrix is different from zero and the control points $\left[\mathbf{p}_{0}, \mathbf{p}_{1}, \mathbf{p}_{n}, \mathbf{p}_{n-1}, \mathbf{q}_{0}, \mathbf{q}_{n+2}\right]$ must be included in the list of the $(n+2)$ fixed control points.

Let $[\operatorname{RDBP}(4,6)]_{Q}$, and from the Equation (18) for $i=0, \ldots, 5$, we find the control points and the centroid point Equation (19) in the form

$$
\left[\left(\mathbf{q}_{6}-\mathbf{q}_{0}\right)-\alpha_{1} \mathbf{v}\right]=\alpha_{0}\left(\mathbf{p}-\mathbf{p}_{0}\right)+\alpha_{2}\left(\mathbf{p}_{5}-\mathbf{p}\right) .
$$

The points $\left[\mathbf{p}_{0}, \mathbf{p}_{1}, \mathbf{p}_{3}, \mathbf{p}_{4}, \mathbf{q}_{0}, \mathbf{q}_{6}\right]$ are fixed and the elected value $\alpha_{1}$ verify Equation (20). Then, by constraint (1), we can determine the centroid point $\mathbf{p}$ in a position likely in the case of $\rho(u)$ linear, i.e., expressing of the formulae (13). The value $\alpha_{0}$ and $\alpha_{1}$ in Equation (21) can be determined by the form (11). After that, we can calculate the control points $\left[\mathbf{q}_{1}, \mathbf{q}_{5}, \mathbf{p}_{2}, \mathbf{q}_{2}, \mathbf{q}_{3}, \mathbf{q}_{4}\right]$ in the equation system (18) of the six equations and case of its determinant of coefficients matrix is different from zero, i.e., $\alpha_{2} \neq \alpha_{0}$, we get

$$
\begin{gathered}
\mathbf{q}_{1}=\left[\mathbf{q}_{0}+\frac{2}{3} \alpha_{0}\left(\mathbf{p}_{1}-\mathbf{p}_{0}\right)\right] ; \quad \mathbf{q}_{5}=\left[\mathbf{q}_{6}-\frac{2}{3} \alpha_{2}\left(\mathbf{p}_{4}-\mathbf{p}_{3}\right)\right] ; \quad \mathbf{p}_{2}=\frac{1}{\left(-6 \alpha_{0}+6 \alpha_{2}\right)}\left[\mathbf{w}_{1}+\mathbf{w}_{2}+\mathbf{w}_{3}+\mathbf{w}_{4}\right] ; \\
\mathbf{q}_{2}=\frac{1}{30}\left[\mathbf{w}_{1}+12 \alpha_{0} \mathbf{p}_{2}\right] ; \quad \mathbf{q}_{3}=\frac{1}{30}\left[\left(12 \alpha_{0}+12 \alpha_{1}+6 \alpha_{2}\right) \mathbf{p}_{2}+\mathbf{w}_{1}+\mathbf{w}_{2}\right] ; \quad \mathbf{q}_{4}=\frac{1}{-30}\left[\mathbf{w}_{4}-12 \alpha_{2} \mathbf{p}_{2}\right] ;
\end{gathered}
$$

where

$$
\begin{aligned}
& \mathbf{w}_{1}=\left[30 \mathbf{q}_{1}-12 \alpha_{0} \mathbf{p}_{2}+4\left(\alpha_{0}+2 \alpha_{1}+\alpha_{2}\right)\left(\mathbf{p}_{1}-\mathbf{p}_{0}\right)\right] \\
& \mathbf{w}_{2}=\left[6 \alpha_{0} \mathbf{p}_{3}-6\left(\alpha_{0}+2 \alpha_{1}+\alpha_{2}\right) \mathbf{p}_{1}+2 \alpha_{2}\left(\mathbf{p}_{1}-\mathbf{p}_{0}\right)\right] ; \\
& \mathbf{w}_{3}=\left[2 \alpha_{0}\left(\mathbf{p}_{4}-\mathbf{p}_{3}\right)+6\left(\alpha_{0}+2 \alpha_{1}+\alpha_{2}\right) \mathbf{p}_{3}-6 \alpha_{2} \mathbf{p}_{1}\right] ; \\
& \mathbf{w}_{4}=\left[-30 \mathbf{q}_{5}+4\left(\alpha_{0}+2 \alpha_{1}+\alpha_{2}\right)\left(\mathbf{p}_{4}-\mathbf{p}_{3}\right)+2 \alpha_{2} \mathbf{p}_{3}\right] .
\end{aligned}
$$

Based on the discussion, results were obtained as follows: The construction method $[\operatorname{RDBP}(n, n+2)]_{Q}$. has many advantages for modeling the shape of regular developable patches. First, it can facilitate the determination of some control points of both curves $\mathbf{C}_{1}(u)$ and $\mathbf{C}_{2}(u)$ to model the curves. Second, by moving the centroid point position $\mathbf{p}$ and changing the parameter value $\alpha_{1}$, it will modify the various forms of the patches. Figure $5 \mathrm{a}, \mathrm{b}$ show the patches shape changing when the position $\mathbf{p}$ is moved down and Figure $5 c, d$ present the patches shape changing when the value $\alpha_{1}=(0.6) . w$ and $\alpha_{1}=(-0.8) . w$. 


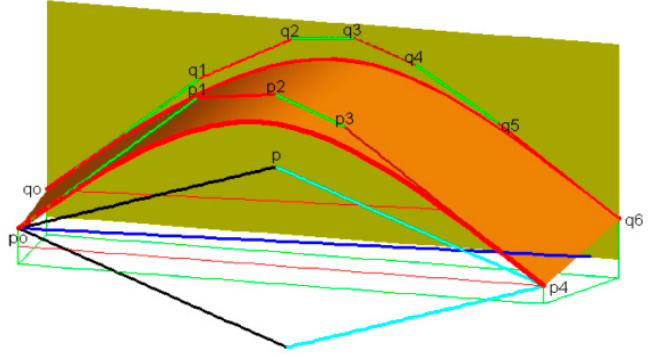

(a)

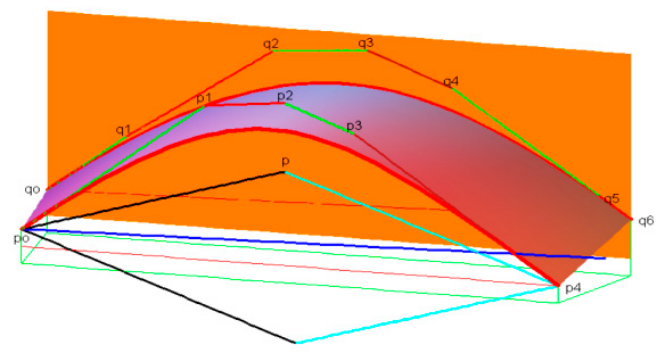

(c)

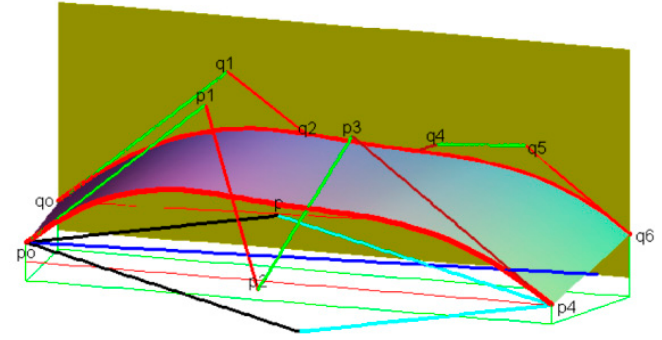

(b)

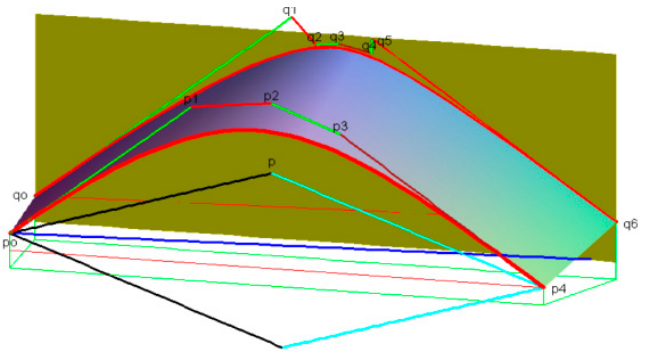

(d)

Figure 5. Construction $[\operatorname{RDBP}(4,6)]_{Q}$. (a) Value $\alpha_{1}=0$; (b) Value $\alpha_{1}=0$ and position $\mathbf{p}$ moved down; (c) Value $\alpha_{1}=(0.6) . w ;$ (d) Value $\alpha_{1}=(-0.8) . w$.

\section{Conclusions}

We developed the method to design a regular developable Bézier patch through two given Bézier curves from the combination of four, five, and six degrees laid respectively in two parallel planes. Using the determination of some Bézier control points of the curves and the parameter fixation of the developable condition, we can control and model various shapes of the Bézier developable patches as follows: The shape $[\operatorname{RDBP}(n, n)]_{C}$ with $\rho(u)$ positive constant is entirely determined by the conditions of the vectors $\left(\mathbf{p}_{n}-\mathbf{p}_{0}\right) / /\left(\mathbf{q}_{n}-\mathbf{q}_{0}\right)$ and some fixed Bézier control points for Bézier curves $\mathbf{C}_{1}(u)$ and $\mathrm{C}_{2}(u)$. The shape modeling $[\operatorname{RDBP}(n, n+1)]_{L}$ with $\rho(u)$ positive linear depends on some fixed Bézier control points and the determination of centroid point position $\mathbf{p}$ of the Bézier polygon of curve $\mathbf{C}_{1}(u)$. Finally, the shape of patches $[\operatorname{RDBP}(n, n+2)]_{Q}$ with $\rho(u)$ positive quadratic can be modeled by the fixation of some control points of the Bézier curves $\mathbf{C}_{1}(u)$ and $\mathbf{C}_{2}(u)$, the modification position of the centroid point $\mathbf{p}$, and the given parameter value $\alpha_{1}$.

Using two curves that lie, respectively, in the plane parallel and the developable surface, criteria can be designed to determine the regular developable Bézier patches of four, five, and six degrees. In further studies, discussions should focus on how to construct the developable patches in which the two boundaries are space curves and can be adjusted by some shape parameters.

Acknowledgments: The author wants to acknowledge the support of Mathematics staff of Jember University for providing this research.

Conflicts of Interest: The author declares no conflict of interest.

\section{References}

1. Aumann, G. Interpolation with developable Bézier patches. CAGD 1991, 23, 409-420. [CrossRef]

2. Frey, W.H.; Bindschadler, D. Computer-Aided Design of a Class of Developable Bézier Surfaces; General Motors R\&D Publication: Detroit, MI, USA, 1993.

3. Chu, C.H.; Séquin, C.H. Developable Bézier patches: Properties and design. CAGD 2002, 34, 511-527. [CrossRef] 
4. Aumann, G. A simple algorithm for designing developable Bézier surfaces. CAGD 2002, 20, 601-616. [CrossRef]

5. Chalfant, J.S. Analysis and Design of Developable Surfaces for Shipbuilding. Master's Thesis, Massachusetts Institute of Technology, Cambridge, MA, USA, 1997.

6. Zhao, H.; Wang, G. A new method for designing a developable surface utilizing the surface pencil through a given curve. Prog. Nat. Sci. 2008, 18, 105-110. [CrossRef]

7. Al-Ghefari, R.A.; Abdel-Baky, R.A. An approach for designing developable surface with a common geodesic curve. Int. J. Contemp. Math. Sci. 2013, 8, 875-891. [CrossRef]

8. Park, F.C.; Yu, Y.; Chun, C.; Ravani, B. Design of developable surfaces using optimal control. Trans. ASME 2002, 124, 602-608. [CrossRef]

9. Elber, G. Approximation algorithm for developable surfaces. CAGD 1995, 16, 539-556.

10. Sun, M.; Fiume, E. A Technique for Constructing Developable Surfaces; University of Toronto: Toronto, ON, Canada, 1996; pp. 176-185.

11. Xu, G.; Rabczuk, T.; Güler, E.; Wu, Q.; Hui, K.; Wang, K. Quasi-harmonic Bézier approximation of minimal surfaces for finding forms of structural membranes. Comput. Struct. 2015, 161, 55-63. [CrossRef]

12. Xu, G.; Li, M.; Mourrain, B.; Rabczuk, T.; Xu, J.; Bordas, S.P.A. Constructing IGA-suitable planar parameterization from complex CAD boundary by domain partition and global/local optimization. Comput. Methods Appl. Mech. Eng. 2017, 328, 175-200. [CrossRef]

13. Bodduluri, R.M.C.; Ravani, B. Design of developable surfaces using duality between plane and point geometries. CAGD 1993, 25, 621-632. [CrossRef]

14. Pottmann, H.; Farin, G. Developable rational Bézier and B-spline surfaces. CAGD 1995, 12, 513-531. [CrossRef]

15. Pottmann, H.; Wallner, J. Model fabrication using surface layout projection. CAD 1999, 27, $283-291$.

16. Hu, G.; Wu, J.L.; Qin, X.Q. A new approach in designing of local controlled developable H-Bézier surfaces. Adv. Eng. Softw. 2018, 121, 26-38. [CrossRef]

17. Hu, G.; Cao, H.X.; Zhang, S.X.; Guo, W. Developable Bézier-like surfaces with multiple shape parameters and its continuity conditions. Appl. Math. Model. 2017, 45, 728-747. [CrossRef]

18. Kusno. Contribution à la Solution du Problème de Construction et de Raccordement Géometrique de Surfaces Développable Régulièrs à L'aide des Carreaux de Bézier. Ph.D. Thesis, Université de Metz, Metz, France, 1998. (In French)

19. Hui, D.W. Etude sur la Représentation des Surfaces Complexes: Application à la Reconstruction de Surfaces Echantillonées. Ph.D. Thesis, Télécom ParisTech (ENST), Paris, France, 1988. (In French) 